\title{
Surgical Management of Liver Metastases from Colorectal Carcinoma
}

\author{
Lou-Anne Acevedo-Moreno, MD ${ }^{1}$ Federico Aucejo, $\mathrm{MD}^{1}$ \\ ${ }^{1}$ Department of General Surgery, Digestive Disease and Surgery \\ Institute, Cleveland Clinic, Cleveland, Ohio \\ Address for correspondence Federico Aucejo, MD, Department of \\ General Surgery, Digestive Disease and Surgery Institute, Cleveland \\ Dig Dis Interv 2017;1:163-170. \\ Clinic, 9500 Euclid Avenue, A100, Cleveland, OH 44195 \\ (e-mail: aucejof@ccf.org).
}

\begin{abstract}
Keywords

- CRLM

- colorectal carcinoma

- colorectal liver metastases

Colorectal carcinoma continues to be a leading cause in cancer-related mortality with more than 130,000 new cases diagnosed annually in the United States. About $50 \%$ of patients will develop colorectal cancer liver metastasis (CRLM). Liver resection continues to be the mainstay therapy in the management of CRLM and is associated with 25 to $60 \% 5$-year survival. Alternative nonsurgical therapies offer modest survival when CRLM is unresectable. Herein, we provide an overview of key aspects of surgical approaches to the treatment of CRLM.
\end{abstract}

Colorectal carcinoma (CRC) represents a leading cause of cancer-related mortality with more than 130,000 new cases diagnosed annually in the United States. ${ }^{1,2}$ Worldwide, CRC is the most common gastrointestinal malignancy affecting nearly 1 million people each year. ${ }^{3}$

The liver is the most frequent site of metastases and approximately $50 \%$ of the patients will experience this complication. ${ }^{4,5}$ While about a third of patients will develop synchronous CRC liver metastasis (CRLM), 25 to $50 \%$ of patients will develop metachronous disease within 3 years after resection of the primary tumor. ${ }^{6}$

Liver resection continues to be the mainstay therapy in the management of CRLM, allowing 25 to 60\% 5-year survival. However, only about a third of patients are amenable to resection at the time of diagnosis and long-term survival in the setting of unresectable CRLM is dismal ( $10 \%$ at 5 years). ${ }^{4}$

Efforts to refine and expand surgical intervention along with evolution of modern systemic therapies are expected to improve further patient survival. Herein, we describe and discuss current surgical techniques and strategies to approach the patient with CRLM, including two-stage hepatectomy, associating liver partition and portal vein ligation for staged hepatectomy (ALPPS), parenchyma-sparing hepatectomy, simultaneous versus staged resection of primary tumor and hepatectomy, colon-first versus liver-first approach for synchronous CRLM, best use of perioperative chemotherapy, use of hepatic artery chemotherapy infusion (HAI) pump, hepa-

received

June 20, 2017

accepted after revision

August 11, 2017

published online

October 17, 2017 tectomy in the setting of R1 resection, hepatectomy in the setting of extrahepatic disease (EHD), and liver transplantation (LT) for unresectable CRLM.

\section{Definition of "Resectable CRLM" Future Liver Remnant}

The main reason to deem CRLM unresectable is insufficient future liver remnant (FLR) volume.

In general, in patients with normal liver function and absence of portal hypertension, performing a resection preserving 20 to $30 \%$ of the whole liver volume is accepted as safe. However, in patients with underlying chronic liver disease or hepatotoxicity from prolonged systemic chemotherapy, FLR should be in the order of 30 to $40 \%$ to avoid complications from liver failure. ${ }^{2,5}$

When planning a major hepatectomy, presurgical liver volume assessment utilizing cross-sectional imaging is considered standard of care. If FLR is insufficient, contralateral portal vein embolization (PVE) will allow FLR hypertrophy in approximately 4 to 6 weeks. $^{5}$

In addition to liver volume assessment, liver function can be addressed by assessing indocyanine green clearance. Indocyanine green is metabolized in the liver and excreted in the bile. Indocyanine green retention $>15 \%$ after 15 minutes of intravenous administration reveals impaired liver function, and therefore large hepatectomy should be carefully considered or avoided. $^{7}$
Copyright $\odot 2017$ by Thieme Medical Publishers, Inc., 333 Seventh Avenue, New York, NY 10001, USA. Tel: +1(212) 584-4662.
DOI https://doi.org/ 10.1055/s-0037-1606827. ISSN 2472-8721.
Guest Editors: Charles Martin III, MD and Indravadan J. Patel, MD 


\section{Surgical Resection}

Ideally, the surgical approach to CRLM with curative intent aims to achieve R0 resection (negative surgical margins) while sparing sufficient liver remnant volume.

The definition of resectability in patients with CRLM has evolved over time. In the past, patients with more than four metastases, bilobar involvement, lesions larger than $5 \mathrm{~cm}$, and the presence of extrahepatic disease were not considered surgical candidates. ${ }^{8}$ In the modern era, with advancement of surgical technique and postoperative care and superior systemic chemotherapy, liver resection can be attempted as long as functional 20 to 30\% FLR is achieved. Furthermore, even if R0 resection is unlikely or if there is oligo-focal extrahepatic disease amenable to resection, surgery is still associated with survival benefit when compared with systemic chemotherapy only. ${ }^{9}$

\section{Two-Staged Hepatectomy}

In the presence of bilobar disease with one lobe carrying most of the tumor burden, a staged hepatectomy is the standard surgical approach if CLRM cannot be cleared in one operation due to insufficient FLR., ${ }^{5,9}$

Typically, the lobe with less disease is cleared by resection or resection and ablation to spare parenchyma during the first stage and this is followed by contralateral PVE or ligation to enhance hypertrophy of the treated lobe. Four to 6 weeks after the PVE, reassessment of the FLR is carried out through cross-sectional imaging and a second-stage resection of the lobe with the larger tumor bulk is performed ${ }^{10}$ (-Figs. 1-3).

The feasibility of performing a two-staged hepatectomy has been reported to be in the order of 60 to $70 \%$ and 5 -year survival in the order of $40 \%$ which is similar to survival in patients undergoing a single-staged hepatectomy. ${ }^{2}$

\section{Associating Liver Partition and Portal Vein Ligation for Staged Hepatectomy}

When PVE fails to achieve sufficient FLR hypertrophy, ALPPS can be considered as an advanced surgical technique to clear CRLM.

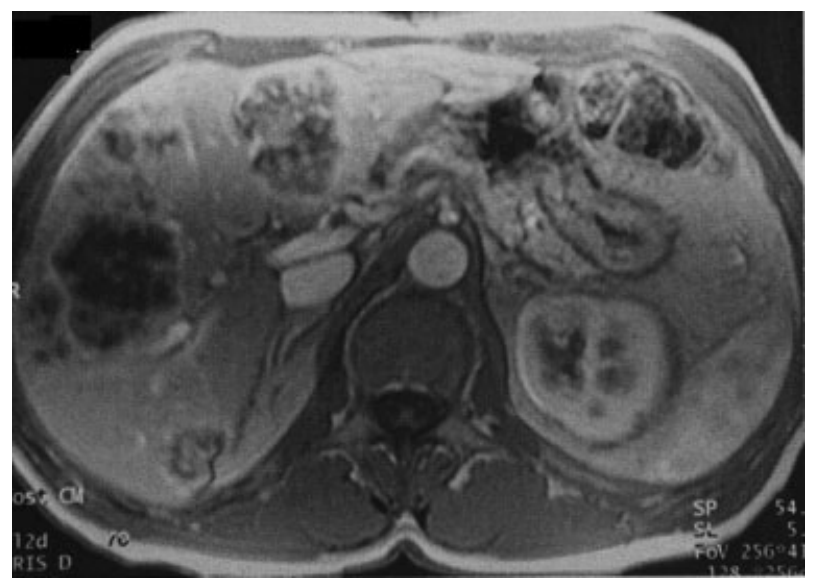

Fig. 1 Two-stage hepatectomy. Bilobar liver metastases.

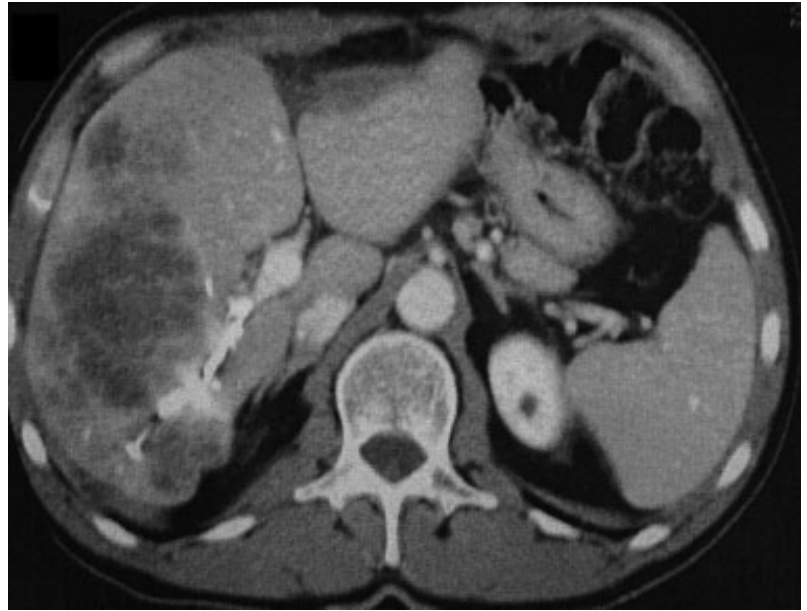

Fig. 2 Two-stage hepatectomy. First stage: resection of metastasis from left lateral segment and right portal vein embolization.

A first-stage intervention consists of clearing the small FLR by resection or ablation, and in situ parenchyma splitting to separate the cleared FLR from the rest of diseased liver. Simultaneously, the portal vein of the lobe affected with the large bulk of disease is ligated. A final staged hepatectomy follows in approximately 10 to 14 days after the initial operation to complete clearance of the disease. ${ }^{5,10}$

The typical ALPPS procedure involves initial treatment of the left lateral segment with partition of parenchyma just to the right of the insertion of the falciform ligament with right PV ligation. This is followed by a second-stage extended right hepatectomy approximately 1 to 2 weeks after the first operation. $^{5,10}$

Estimated liver volume growth after PVE is in the order of $2 \%$ per week, while after ALPPS it is in the order of 10 to $20 \%$. Therefore, successful rapid hypertrophy of FLR could be achieved with ALPPS in 1 to 2 weeks.,11 The physiologic explanation for the rapid and enhanced liver growth is related to disruption of interlobar hepatic vascular connections in addition to PV ligation which re-routes inflow with growth factors exclusively to the FLR. ${ }^{10}$

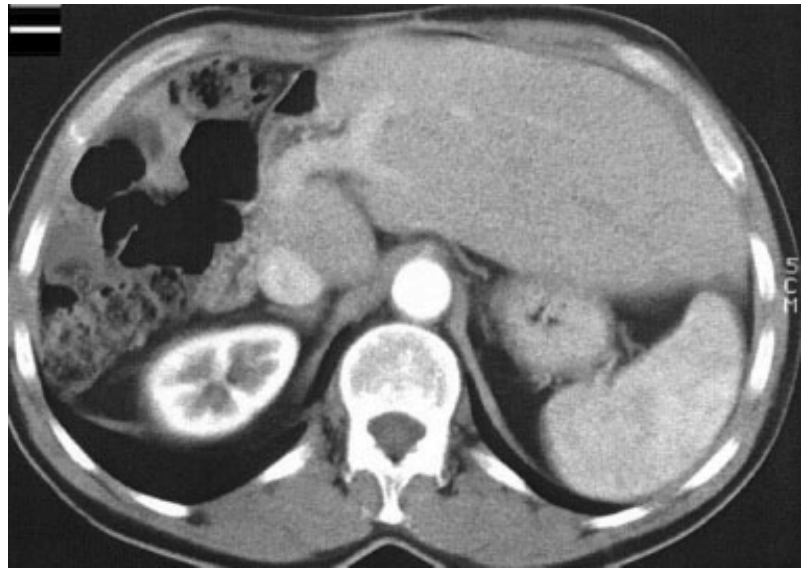

Fig. 3 Two-stage hepatectomy. Extended right hepatectomy. Post right portal vein embolization hypertrophy of left lateral segment. 
Although published data have reported over 90\% feasibility of completion of the two-staged hepatectomy cycle in ALPPS procedures, and approximately $90 \%$ achievement of R0 resection, morbidity and mortality rates have been higher than associated with standard two-staged hepatectomy or single hepatectomy procedures. ${ }^{12}$

A meta-analyses by Schadde et $\mathrm{al}^{12}$ revealed a high feasibility rate (97\%), high $\mathrm{R} 0$ resection rate $(91 \%)$, and comparable but higher morbidity (44\%) and mortality (11\%) compared with conventional two-stage hepatectomies. Recurrence-free survival (RFS) was also significantly lower in ALPPS patients. Careful patient selection should be carried out when attempting ALPPS, and should be considered when PVE fails or is unlikely to achieve sufficient FLR.

\section{Parenchyma-Sparing Hepatectomy: The Transparenchymal Approach}

As opposed to hepatocellular carcinoma where anatomic liver resection has shown superior results compared with nonanatomic resection, wedge or nonanatomic liver resection for CRLM is sufficient as long as surgical margins are negative. ${ }^{3}$ Furthermore, although it has been reported that a negative surgical margin of at least $1 \mathrm{~cm}$ is associated with superior results, even in the presence of $\mathrm{R} 1$ resection survival is better compared with no resection. ${ }^{13}$ This allows for surgical strategies to spare parenchyma when deemed necessary. Although ablation of small metastases can be implemented to spare parenchyma and produce comparable results, resection should always be opted first, if possible. Such is the case of unifocal or oligo-focal CRLM $<5 \mathrm{~cm}$ deeply located in the parenchyma. The transparenchymal approach entails marking the boundaries of the tumor with needles placed under ultrasound guidance. Meticulous transparenchymal dissection using the ultrasonic dissector allows to reach the deeply located metastases preserving liver volume (-Figs. 4-6). As CRLMs are likely to recur and repeat hepatectomy required, parenchyma preserving resection

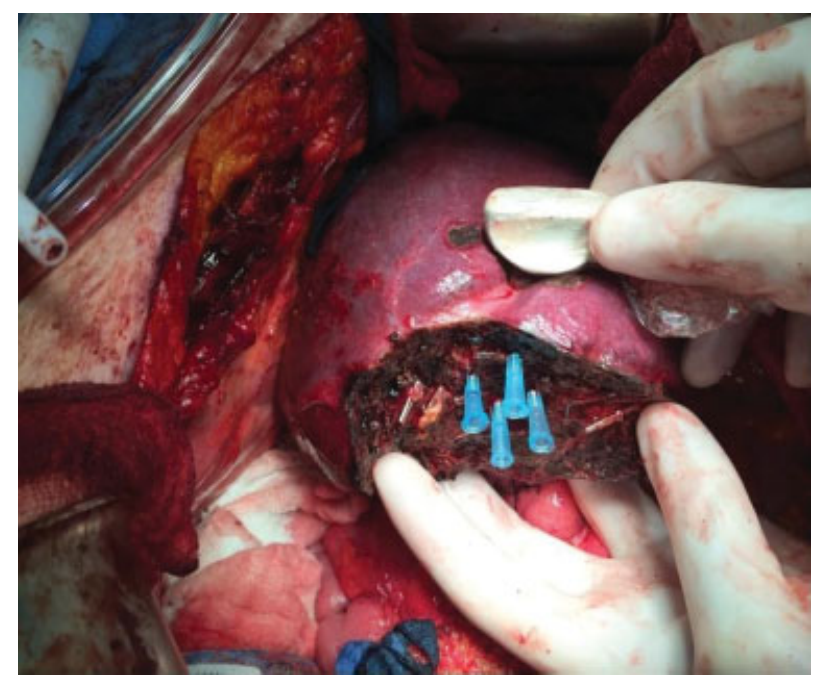

Fig. 4 Ultrasound-guided marking for parenchyma sparing. Transparenchymal approach of liver metastases.

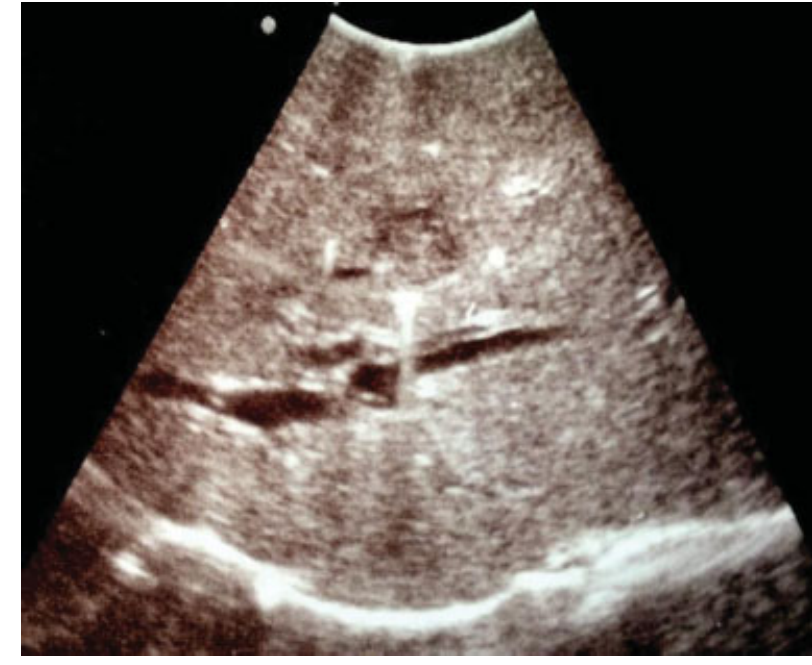

Fig. 5 Ultrasound-guided transparenchymal approach.

strategies such as the transparenchymal approach should be considered.

\section{Simultaneous versus Staged Resection for Synchronous CRLM}

Synchronous liver metastases occur in approximately $25 \%$ of patients with CRC. ${ }^{8}$ In general, it is accepted that simultaneous resection of primary CRC and hepatectomy should be performed when liver resection is only minor. ${ }^{1}$ On the other hand, if a major hepatectomy is needed, simultaneous resection should be discouraged and instead a staged resection should be considered. ${ }^{1,2}$

The rationale for this is twofold. In the presence of advanced liver disease, it is recommended to proceed with systemic chemotherapy after removal of the primary tumor to assess tumor biology and rule out progression of disease beyond the liver. Additionally, extensive hepatectomy performed simultaneously with colorectal surgery is associated with increased complications including sepsis and liver failure. ${ }^{14}$

Some authors, however, have proposed the opposite. In a study by Abelson et al, ${ }^{1}$ simultaneous surgery was associated

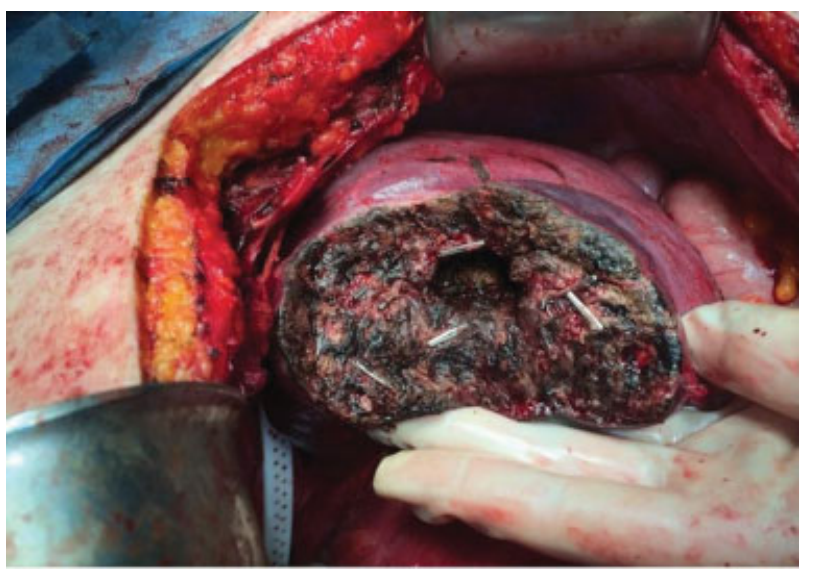

Fig. 6 Resection bed. Transparenchymal approach. 
with reduced health care utilization, reduced readmission rate, shorter hospital stay, and lower costs. In this study, a total of 1,088 patients underwent simultaneous resection for synchronous CRC and CRLM between 2005 and 2014. Twenty-nine percent of patients undergoing simultaneous resection received lower anterior resections and $4.4 \%$ received abdominoperineal resections versus 25 and 6\% in the group undergoing staged resection. In regard to hepatectomy, 85\% received partial hepatectomy and $14 \%$ received total lobectomy in the simultaneous resection group versus $69 \%$ partial hepatectomy and $31 \%$ total lobectomy in the staged resection group. ${ }^{1}$ As reported, it has to be underscored that the numbers of patients undergoing total lobectomy during staged approach were double than the patients undergoing total lobectomy during simultaneous approach.

In a study by Adam et $\mathrm{al}^{15}$ it was concluded that simultaneous resection should be discouraged when major hepatectomy is required (involving three hepatic segments or more), or when complex rectal surgery is to be performed, due to significantly higher postoperative mortality and morbidity. Similarly, other studies recommended avoiding simultaneous approach when synchronous CRLMs are noted on exploration for emergency situations such as colonic perforation, bowel obstruction, or bleeding, especially in patients with increased risk of postoperative liver failure (chronic liver disease) or in patients with insufficient FLR. ${ }^{16}$

Careful patient selection (age, performance status, comorbidities) and tumor biology assessment should be evaluated when considering simultaneous resection of primary tumor and large hepatectomy.

\section{Colon-First versus Liver-First Approach for Synchronous CRLM}

The traditional approach to synchronous CRLM is to treat the CRC first and after a course of systemic chemotherapy, proceed with hepatectomy. However, there are some authors who have proposed the liver-first approach. The underlying rationale is to prevent progression of advanced CRLM to unresectable stage which may occur while colorectal surgery is undertaken. ${ }^{17-21}$

The liver-first approach comprises of preoperative chemotherapy followed by liver resection and subsequent resection of the primary tumor as a staged procedure. The liver-first approach was first reported in 2006, with 5-year overall survival (OS) of 40 to $50 \%$ and disease-free survival of 68 and $30 \%$ at 1 and 3 years, respectively. ${ }^{17}$

A few studies evaluating outcomes of patients undergoing the liver-first approach reported morbidity in the range of 11 to $37 \%$ and postoperative mortality less than $4 \%$. Three and 5 -year OS rates were 41 to $79 \%$ and 31 to $39 \%$, respectively, and disease recurrence rates were in the order of 25 to $70 \%$.

In a study by De Rosa et al, ${ }^{19}$ comparing outcomes of patients who underwent liver-first approach, it was concluded that this strategy can be considered preferable for patients with early-stage rectal cancer and extensive liver disease.

\section{Best Use of Perioperative Chemotherapy}

Neoadjuvant chemotherapy when performing liver resection for CRLM should be used cautiously. The larger number of chemotherapy lines and cycles correlates with increased liver toxicity and risk of tumor chemoresistance. This jeopardizes tolerance to hepatectomy and increases risk of postsurgical tumor recurrence decreasing altogether postsurgical survival. ${ }^{8}$

Another caveat associated with presurgical chemotherapy especially in the setting of small CRLM is that disease can vanish (vanishing metastasis), and therefore can be difficult or not possible to be found during surgical exploration. ${ }^{8}$

The incidence of disappearing liver metastases varies from 7 to $24 \%$, and its management is challenging because complete radiologic response does not necessarily correlate with pathological response. ${ }^{22}$

In line with this, Passot et $\mathrm{al}^{23}$ evaluated the placement of fiducials in patients undergoing hepatic resection of CRLM. The indication was for metastases at risk of disappearing to aid in intraoperative localization during parenchymal-sparing hepatectomy. Fiducial markers were placed in metastases $<20 \mathrm{~mm}$ in size and located $>10 \mathrm{~mm}$ deep in the liver parenchyma. No local recurrences were observed in sites treated after fiducial placement. ${ }^{23}$

To avoid vanishing metastases, the length of neoadjuvant chemotherapy should be determined based on tumor response (assessment of tumor biology) and to the point when disease becomes downstaged to resectable, not to the point of complete radiologic response. ${ }^{8}$

\section{Significance of Surgical Margin in CRLM}

A positive surgical margin increases the risk of local recurrence and compromises long-term survival. ${ }^{2,8,13}$ Sadot et $\mathrm{al}^{24}$ evaluated 2,368 patients undergoing hepatic resection for CRLM, noticing that increasing margin width was associated with improved survival (5-year OS: 46 and $48 \%$ for patients undergoing R0 resection with $1-9 \mathrm{~mm}$ margins and $10 \mathrm{~mm}$ or greater margins, respectively; compared with 24 and $26 \%$ for patients undergoing $\mathrm{R} 1$ resection and $\mathrm{R} 0$ resection with submillimeter margins, respectively).

The EGOSLIM group (an international multidisciplinary group of experts in managing CRLM that convened to discuss synchronous metastases and their management) concluded that even though safe resection margins are still a goal of therapy, minimal surgical clearance margin of $1 \mathrm{~mm}$ can be considered sufficient. ${ }^{15}$ In addition to surgical technique, this underscores the importance of the underlying tumor biology impacting patient survival. In line with this, while R0 resection with wide margins is the preferred outcome, an anticipated R1 resection should not be considered a contraindication to resection. ${ }^{25}$

\section{Hepatic Arterial Chemotherapy Infusion}

Directed chemoinfusion to the liver through a surgically implantable pump is an adjunct therapy option that can convert unresectable to resectable disease, can deliver superior local 
tumor control, can prevent disease recurrence, and can improve patient survival. ${ }^{25}$ The rationale for regional hepatic arterial infusion is that CRLMs are perfused by the hepatic artery. Utilized chemotherapy agent (floxuridine) is extracted by the liver during first pass and thus achieving higher and more effective concentrations with no or less systemic toxicity. ${ }^{26}$

HAI can be implemented in both neoadjuvant and adjuvant settings and in combination with systemic chemotherapy. ${ }^{8}$ Adjuvant intra-arterial chemotherapy in combination with systemic chemotherapy has been beneficial in prolonging time to recurrence and preventing hepatic recurrence after resection of CRLM. ${ }^{27}$ Results of studies reporting perioperative use of HAI are summarized in - Table 1.

In a Memorial Sloan Kettering Cancer Center study including 2,368 patients undergoing complete resection for CRLM, the median survival of patients who received HAI ( $n=785$ [653 adjuvant; 132 neoadjuvant]) was 67 months compared with 47 months for patients who did not receive HAI $(n=1,583){ }^{26}$

Zampino et $\mathrm{al}^{28}$ reported a response rate of $92 \%$ with median survival of 50.8 months, in 49 patients undergoing HAI in the neoadjuvant setting, combined with systemic oxaliplatin and irinotecan.

Ammori et $\mathrm{al}^{29}$ evaluated 373 patients with unresectable CRLM treated with a combination of HAI and systemic chemotherapy, reporting $25 \%$ of conversion rate undergoing complete resection and/or ablation, with a median OS of 59 months and predicted 5 -year survival of $47 \%$ compared with 16 months and $6 \%$, respectively, for those who did not have surgery.

House et $\mathrm{al}^{30}$ made a comparison of adjuvant HAI-Floxuridine (FUDR) in combination with systemic chemotherapy (oxaliplatin or irinotecan) versus systemic chemotherapy alone. This study revealed that for the adjuvant HAI + systemic chemotherapy, the 5-year liver RFS, RFS, and OS were 75, 48, and $79 \%$, respectively, compared with 55,25 , and $55 \%$, respectively, in the adjuvant systemic chemotherapy alone group.

- Figs. 7 to 9 demonstrate radiologic tumor response to liver resection and hepatic artery chemoinfusion using FUDR.

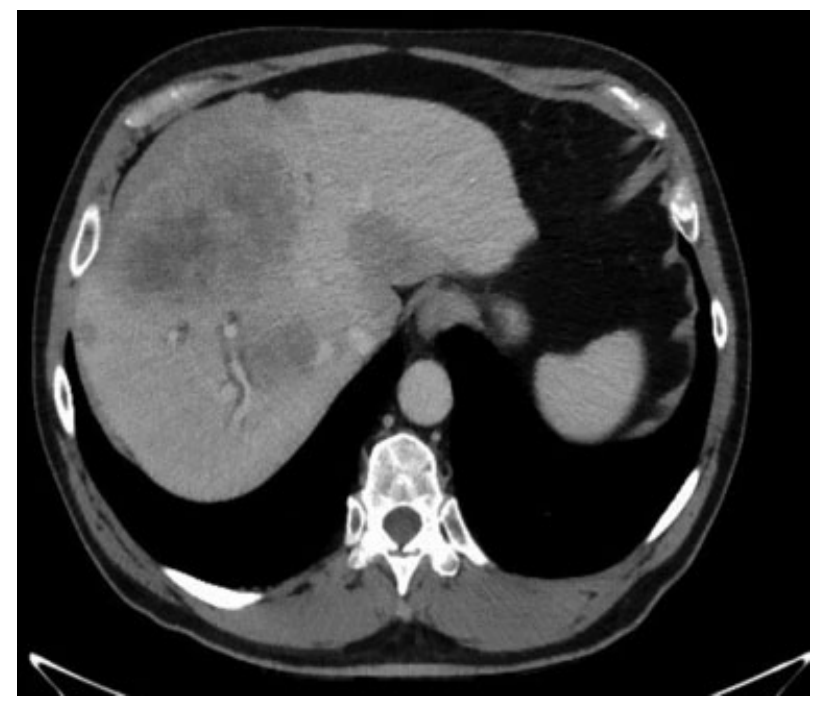

Fig. 7 Advanced colorectal cancer liver metastasis before liver resection and hepatic artery pump chemoinfusion.

\section{Hepatectomy in the Setting of Extrahepatic Disease}

EHD is associated with poor survival, and surgical treatment should only be considered in patients with oligo-focal EHD disease and optimal response to systemic therapy. ${ }^{8}$

In a literature review by Quan et al, ${ }^{31}$ outcomes in patients who had liver and lung metastases, liver and portal node metastases, and liver and other extrahepatic diseases were reported in 14, 10, and 14 studies, respectively. Timing of resection of liver and pulmonary metastases varied in the studies and included simultaneous hepatectomy and pulmonary metastatectomy, hepatectomy followed by pulmonary metastatectomy, and pulmonary metastatectomy followed by hepatectomy. In this review, it was concluded that select patients with pulmonary and CRLM may benefit from resection, with an estimated 5 -year survival of $49 \%$ for patients with lung metastases appearing after liver metastases.

Table 1 Summary of studies reporting perioperative HAI

\begin{tabular}{|c|c|c|c|}
\hline Authors & HAI modality & $\begin{array}{l}\text { Median } \\
\text { overall survival }\end{array}$ & $\begin{array}{l}\text { Hepatic } \\
\text { recurrence-free survival }\end{array}$ \\
\hline Zampino et al ${ }^{28}$ & Neoadjuvant $\mathrm{HAI}+$ systemic Ox and Iri & $39.8 \mathrm{mo}$ & $\mathrm{N} / \mathrm{A}$ \\
\hline Ammori et al ${ }^{29}$ & Neoadjuvant $\mathrm{HAI}+$ bevacizumab & $59 \mathrm{mo}$ & $47 \%$ at $5 y$ \\
\hline Groot Koerkamp et al ${ }^{26}$ & $\begin{array}{l}\text { Neoadjuvant HAI } \\
\text { Adjuvant } \mathrm{HAI}\end{array}$ & $\begin{array}{l}46 \mathrm{mo} \\
71 \mathrm{mo}\end{array}$ & $\begin{array}{l}39 \% \\
56 \% \\
\text { At } 5 \text { y }\end{array}$ \\
\hline House et $\mathrm{al}^{30}$ & Adjuvant HAI-FUDR + systemic Ox and Iri & $\begin{array}{l}\text { Ox } 32 \text { mo } \\
\text { Iri } 34 \text { mo }\end{array}$ & $\begin{array}{l}69 \% \\
70 \% \\
\text { At } 5 \text { y }\end{array}$ \\
\hline Ducreux et al ${ }^{36}$ & $\mathrm{HAI}+$ systemic FU & $27 \mathrm{mo}$ & $55 \%$ at 2 y \\
\hline Alberts et $\mathrm{al}^{27}$ & Adjuvant HAI-FUDR + systemic Ox and capecitabine & $32.7 \mathrm{mo}$ & $75.5 \%$ at $2 y$ \\
\hline Kemeny et $\mathrm{al}^{37}$ & HAI-FUDR + systemic FU & $63.7 \mathrm{mo}$ & $90 \%$ at 2 y \\
\hline
\end{tabular}

Abbreviations: FUDR, floxuridine; HAI, hepatic artery chemotherapy infusion; Iri, irinotecan; N/A, not available; Ox, oxaliplatin. 


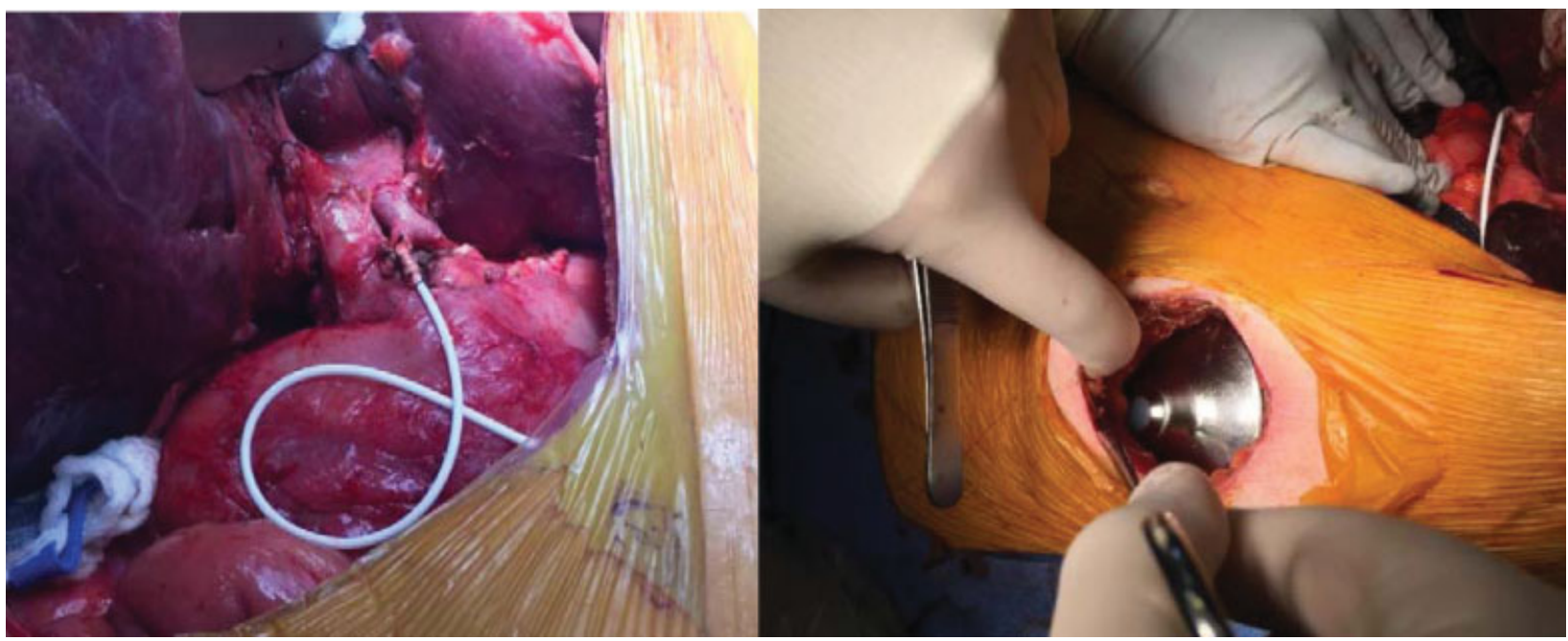

Fig. 8 Hepatic artery pump placement for liver-directed chemoinfusion.

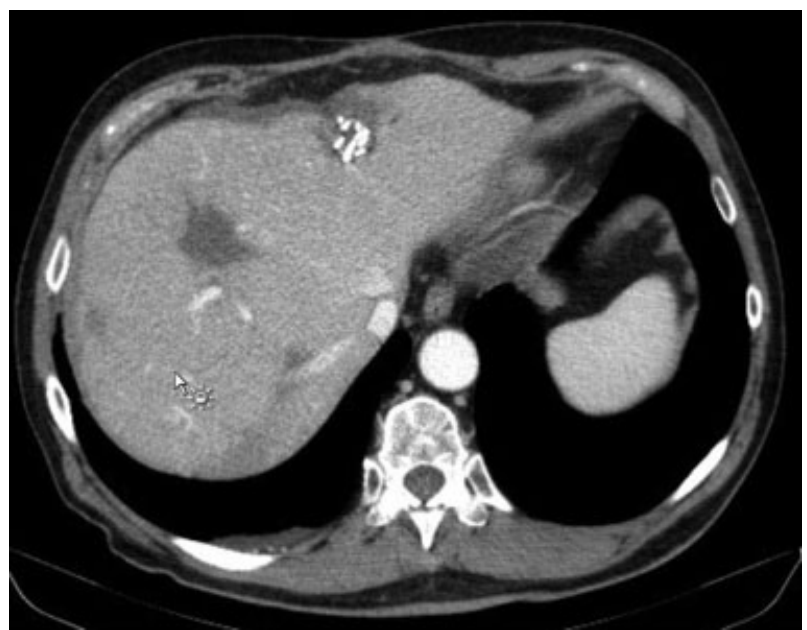

Fig. 9 Radiologic tumor response to liver resection and hepatic artery chemoinfusion.

Elias et al ${ }^{32}$ reviewed 127 patients with radiographically or intraoperatively detected EHD, which represented $8.5 \%$ of 1,359 of patients who underwent hepatic resections for CRLM. Patients with EHD had worse 5-year survival (26\%) than those without EHD (49\%). Also, survival was better for patients with lung, ovarian, and limited peritoneal diseases, and worse for patients with lymph node involvement.
In a study by Pulitanò et $\mathrm{al}^{33}$ out of 1,629 patients undergoing resection for CRLM, 171 had EHD treated with resection. Median OS for these patients was 39 months, with a stratification as follows: lung (46 months), peritoneum (32 months), hepatic pedicle lymph nodes (29 months), and aortocaval lymph nodes (13 months), concluding that the location of EHD seemed to be associated with prognosis. Based on the study's findings, the authors concluded that patients with small number of liver metastasis and single-site EHD (specially lung) should be given consideration for surgery.

\section{Liver Transplantation for Unresectable CRLM}

Liver transplantation for unresectable CRLM was first attempted during the 1980s and early 1990s; however, outcomes were poor and this indication was considered a contraindication. Retrospectively, patient selection was suboptimal and significant morbidity and mortality related not to malignancy but to perioperative complications. ${ }^{17}$ In a recent study from the University of Norway, 21 patients undergoing LT for unresectable CRLM exhibited approximately 60\% 5-year posttransplant survival compared with historic $10 \% 5$-year survival in patients with unresectable liver disease. ${ }^{34}$

Factors that predicted better survival were pre-LT CEA level $<80 \mu \mathrm{g} / \mathrm{L}$, maximum tumor diameter $<5.5 \mathrm{~cm}$, LT at

Table 2 Inclusion/exclusion criteria for LT and risk predictor factors (University of Norway)

\begin{tabular}{|l|l|l|}
\hline Inclusion & Exclusion & Favorable predictive factors \\
\hline $\begin{array}{l}\text { Resection of primary } \\
\text { tumor }\end{array}$ & Weight loss $>10 \%$ & Diameter of the largest CRLM $<5 \mathrm{~cm}$ \\
\hline ECOG 1 OR 0 & Other malignancies & Interval $>2$ y between colorectal and transplant operations \\
\hline $\begin{array}{l}\text { Minimum of 6 wk of } \\
\text { chemotherapy }\end{array}$ & Standard contraindications for LT & Pre-LT carcinoembriogenic antigen level $<80 \mathrm{ng} / \mathrm{mL}$ \\
\hline No extrahepatic disease & BRAF mutation & Responsive or stable disease to chemotherapy \\
\hline
\end{tabular}

Abbreviations: CRLM, colorectal cancer liver metastasis; ECOG, Eastern Cooperative Oncology Group; LT, liver transplantation. 
least 2 years after resection of primary tumor, stable disease, and partial response to neoadjuvant chemotherapy. ${ }^{35}$ In regard to recurrent patterns, those patients who exhibited single-site recurrence in the lung and amenable to resection had better survival than patients with liver recurrence that tended to be large and nonamenable to resection ${ }^{8}$ (see - Table 2 ).

Liver transplantation can become an accepted indication for unresectable CRLM in the near future. Improved patient selection and better understanding of tumor biology along with development of effective systemic therapies will play a pivotal role when considering this option.

\section{Summary}

CRC continues to be a prevalent and deadly disease with approximately $50 \%$ of patients developing metastases, most of which affect the liver. Management of CRLM has evolved over time allowing increased survival. Advanced surgical techniques remain the mainstay of therapy and that along with improved postoperative care and evolution of systemic therapies will likely continue expanding life span of affected patients.

\section{References}

1 Abelson JS, Michelassi F, Sun T, et al. Simultaneous resection for synchronous colorectal liver metastasis: the new standard of care? J Gastrointest Surg 2017;21(06):975-982

2 Dhir M, Sasson AR. Surgical management of liver metastases from colorectal cancer. J Oncol Pract 2016;12(01):33-39

3 Lalmahomed ZS, Ayez N, van der Pool AE, Verheij J, IJzermans JN, Verhoef $C$. Anatomical versus nonanatomical resection of colorectal liver metastases: is there a difference in surgical and oncological outcome? World J Surg 2011;35(03):656-661

4 de Ridder JA, van der Stok EP, Mekenkamp LJ, et al. Management of liver metastases in colorectal cancer patients: a retrospective case-control study of systemic therapy versus liver resection. Eur J Cancer 2016;59:13-21

5 Treska V. Methods to increase future liver remnant volume in patients with primarily unresectable colorectal liver metastases: current state and future perspectives. Anticancer Res 2016;36(05): 2065-2071

6 Hashimoto M, Kobayashi T, Ishiyama K, et al. Efficacy of repeat hepatectomy for recurrence following curative hepatectomy for colorectal liver metastases: a retrospective cohort study of 128 patients. Int J Surg 2016;36(Pt A):96-103

7 Dooley J. Sherlock's Diseases of the Liver and Biliary System. 12th ed. London, UK: Wiley-Blackwell; 2011:681-703

8 Smith JJ, D'Angelica MI. Surgical management of hepatic metastases of colorectal cancer. Hematol Oncol Clin North Am 2015;29(01): 61-84

9 Maroulis I, Karavias DD, Karavias D. General principles of hepatectomy in colorectal liver metastases. Tech Coloproctol 2011; 15(01, Suppl 1):S13-S16

10 Torres OJ, Moraes-Junior JM, Lima e Lima NC, Moraes AM. Associating liver partition and portal vein ligation for staged hepatectomy (ALPPS): a new approach in liver resections. Arq Bras Cir Dig 2012;25(04):290-292

11 McNally SJ, Parks RW. Surgery for colorectal liver metastases. Dig Surg 2013;30(4-6):337-347

12 Schadde E, Schnitzbauer AA, Tschuor C, Raptis DA, Bechstein WO, Clavien PA. Systematic review and meta-analysis of feasibility, safety, and efficacy of a novel procedure: associating liver partition and portal vein ligation for staged hepatectomy. Ann Surg Oncol 2015;22(09):3109-3120
13 Bhutiani N, Philips P, Martin RC II, Scoggins CR. Impact of surgical margin clearance for resection of secondary hepatic malignancies. J Surg Oncol 2016;113(03):289-295

14 Hamed OH, Bhayani NH, Ortenzi G, et al. Simultaneous colorectal and hepatic procedures for colorectal cancer result in increased morbidity but equivalent mortality compared with colorectal or hepatic procedures alone: outcomes from the National Surgical Quality Improvement Program. HPB 2013;15 (09):695-702

15 Adam R, de Gramont A, Figueras J, et al; of the EGOSLIM (Expert Group on OncoSurgery management of LIver Metastases) group. Managing synchronous liver metastases from colorectal cancer: a multidisciplinary international consensus. Cancer Treat Rev 2015;41(09):729-741

16 Reddy SK, Pawlik TM, Zorzi D, et al. Simultaneous resections of colorectal cancer and synchronous liver metastases: a multiinstitutional analysis. Ann Surg Oncol 2007;14(12):3481-3491

17 Collins D, Chua H. Contemporary surgical management of synchronous colorectal liver metastases. F1000 Res 2017;6:598

18 Ihnát P, Vávra P, Zonča P. Treatment strategies for colorectal carcinoma with synchronous liver metastases: which way to go? World J Gastroenterol 2015;21(22):7014-7021

19 De Rosa A, Gomez D, Brooks A, Cameron IC. "Liver-first" approach for synchronous colorectal liver metastases: is this a justifiable approach? J Hepatobiliary Pancreat Sci 2013;20(03): 263-270

20 Giuliante F, Ardito F, Vellone M, et al. Role of the surgeon as a variable in long-term survival after liver resection for colorectal metastases. J Surg Oncol 2009;100(07):538-545

21 Lykoudis PM, O'Reilly D, Nastos K, Fusai G. Systematic review of surgical management of synchronous colorectal liver metastases. Br J Surg 2014;101(06):605-612

22 Kassahun WT. Unresolved issues and controversies surrounding the management of colorectal cancer liver metastasis. World J Surg Oncol 2015;13:61

23 Passot G, Odisio BC, Zorzi D, et al. Eradication of missing liver metastases after fiducial placement. J Gastrointest Surg 2016; 20(06):1173-1178

24 Sadot et al. Resection margin and survival in 2368 patients undergoing hepatic resection for metastatic colorectal cancer: surgical technique or biologic surrogate?Ann Surg 2015;262(03): 476-485

25 Andres A, Majno P, Terraz S, et al. Management of patients with colorectal liver metastasis in eleven questions and answers. Expert Rev Anticancer Ther 2016;16(12):1277-1290

26 Groot Koerkamp B, Sadot E, Kemeny NE, et al. Perioperative hepatic arterial infusion pump chemotherapy is associated with longer survival after resection of colorectal liver metastases: a propensity score analysis. J Clin Oncol 2017;35(17): 1938-1944

27 Alberts SR, Roh MS, Mahoney MR, et al. Alternating systemic and hepatic artery infusion therapy for resected liver metastases from colorectal cancer: a North Central Cancer Treatment Group (NCCTG)/ National Surgical Adjuvant Breast and Bowel Project (NSABP) phase II intergroup trial, N9945/CI-66. J Clin Oncol 2010; 28(05):853-858

28 Zampino MG, Magni E, Ravenda PS, et al. Treatments for colorectal liver metastases: a new focus on a familiar concept. Crit Rev Oncol Hematol 2016;108:154-163

29 Ammori JB, Kemeny NE, Fong Y, et al. Conversion to complete resection and/or ablation using hepatic artery infusional chemotherapy in patients with unresectable liver metastases from colorectal cancer: a decade of experience at a single institution. Ann Surg Oncol 2013;20(09):2901-2907

30 House MG, Kemeny NE, Gönen M, et al. Comparison of adjuvant systemic chemotherapy with or without hepatic arterial infusional chemotherapy after hepatic resection for metastatic colorectal cancer. Ann Surg 2011;254(06):851-856 
31 Quan D, Gallinger S, Nhan C, et al; Surgical Oncology Program at Cancer Care Ontario. The role of liver resection for colorectal cancer metastases in an era of multimodality treatment: a systematic review. Surgery 2012;151(06):860-870

32 Elias D, Liberale G, Vernerey D, et al. Hepatic and extrahepatic colorectal metastases: when resectable, their localization does not matter, but their total number has a prognostic effect. Ann Surg Oncol 2005;12(11):900-909

33 Pulitanò C, Bodingbauer M, Aldrighetti L, et al. Liver resection for colorectal metastases in presence of extrahepatic disease: results from an international multi-institutional analysis. Ann Surg Oncol 2011;18(05):1380-1388

34 Elias D, Viganò L, Orsi F, et al. New perspectives in the treatment of colorectal metastases. Liver Cancer 2016;6(01):90-98
35 Ravaioli M, Ercolani G, Neri F, et al. Liver transplantation for hepatic tumors: a systematic review. World J Gastroenterol 2014; 20(18):5345-5352

36 Ducreux M, Ychou M, Laplanche A, et al; Gastrointestinal Group of the Federation Nationale des Centres de Lutte Contre le Cancer. Hepatic arterial oxaliplatin infusion plus intravenous chemotherapy in colorectal cancer with inoperable hepatic metastases: a trial of the gastrointestinal group of the Federation Nationale des Centres de Lutte Contre le Cancer. J Clin Oncol 2005;23(22):4881-4887

37 Kemeny MM, Adak S, Gray B, et al. Combined-modality treatment for resectable metastatic colorectal carcinoma to the liver: surgical resection of hepatic metastases in combination with continuous infusion of chemotherapy-an intergroup study. J Clin Oncol 2002;20(06):1499-1505 